\title{
Research
}

\section{Underdosing of antiretrovirals in UK and Irish children with HIV as an example of problems in prescribing medicines to children, 1997-2005: cohort study}

Esse N Menson, A Sarah Walker, Mike Sharland, Carole Wells, Gareth Tudor-Williams, F Andrew I Riordan, E G Hermione Lyall, Diana M Gibb, for the collaborative HIV paediatric study steering committee

\begin{abstract}
Objective To measure the extent of underdosing of antiretroviral drugs in children.

Design Multicentre cohort study.

Setting Clinical centres in hospitals in the United Kingdom and Ireland in the collaborative HIV paediatric study (CHIPS).

Participants 615 HIV infected children aged 2-12 years receiving antiretrovirals.

Main outcome measures Doses relative to weight and height compared with current recommended doses in 2004 European guidelines.

Results The CHIPS cohort of 934 children comprises $80 \%$ of diagnosed HIV infected children in the UK and Ireland between January 1997 and March 2005, of which 66\% (615) aged 2-12 years were prescribed antiretrovirals. Actual doses standardised to weight or surface area varied widely across individual drugs, antiretroviral class, and calendar time, with children underdosed (prescribed less than $90 \%$ of current recommended doses) from $6-62 \%$ child time at risk. Three serious issues in prescribing antiretrovirals, which may also be relevant to paediatric prescribing in general, were identified. Firstly, dosing was inadequate before incorrect recommendations at licensing were later revised when important pharmacokinetic results emerged. Secondly, guidelines stating dosage alternatives (by weight/surface area) for the same drug led to different and inconsistent doses. And, thirdly, ongoing growth was not adjusted for.

Conclusions Largely inadvertently, HIV infected children in the United Kingdom and Ireland have been underdosed with antiretrovirals, highlighting problems applicable throughout paediatric prescribing.
\end{abstract}

The evidence base for prescribing to children is poor. Most drugs have limited paediatric pharmacokinetic and pharmacodynamic data, partly due to a longstanding culture of resistance to enrolling children in clinical trials ${ }^{12}$ and the genuine difficulties of undertaking paediatric pharmacokinetic studies. Without age specific data, adult doses are often extrapolated without appropriate regard for age related differences in drug handling or requirements for effectiveness. Lack of acceptable formulations limits the precision with which doses can be prescribed to children as they grow. Many liquids are unpalatable, and doses for older children can be too large in volume. Postmarketing pharmacovigilance of most drugs licensed for children is limited at best, without legal obligation to monitor drugs prescribed off label $\left(25 \%\right.$ of drugs used in paediatric wards $\left.{ }^{3}\right)$. The impact of any deficits in prescribing is unknown.

Legislation from the European Union ${ }^{4}$ and the US Food and Drug Administration, ${ }^{5}$ as well as proposals from the UK Department of Health ${ }^{6}$ aim to tackle such issues and improve paediatric prescribing. Few published studies describe the scale or nature of the obstacles to accurate and effective paediatric prescribing or attempt to identify their causes, yet such studies are essential if interventions are to be appropriately targeted. Antiretroviral prescribing to HIV infected children is a good example of some universal problems.

\section{Methods}

The UK and Irish collaborative HIV paediatric study (CHIPS; www.ctu.mrc.ac.uk/studies/chips.asp) collects clinical, laboratory, and drug information from HIV infected children under the care of specialist or general paediatricians in 23 centres in the United Kingdom and Ireland, ${ }^{7}$ representing $80 \%$ of all known HIV infected children reported to the national study of HIV in pregnancy and childhood. ${ }^{8}$ We analysed each dose of antiretroviral prescribed after January 1997 relative to the most recent height and weight measurement, using the formula surface area $=$ $\sqrt{ }($ weight $(\mathrm{kg}) \times$ height $(\mathrm{cm}) \div 3600)$. We compared the total daily dose with the current recommended dose (CRD; see table A on bmj.com) defined according to 2004 PENTA guidelines, ${ }^{9}$ to evaluate prescribing relative to current best practice (rather than audit against information available at the time of prescribing). We compared the adequacy of dose in three time periods1997-9, the initial era when effective treatment became available for children; 2000-2, after results of paediatric pharmacokinetic studies and European prescription guidelines were published ${ }^{10}$; and from 2003 to March 2005. We focussed on the duration of underdosing between ages 2 and 12 years inclusive because drug pharmacokinetics differ substantially in infancy and adolescence. $^{11} 12$

\section{Results}

Of 934 children in the CHIPS cohort, 615 (66\%) aged 2-12 years were prescribed antiretrovirals (tables 1, 2, and 3). Subsequent analysis excluded 17 children (3\%) from five centres that did not

Table A, showing the recommended doses, and a list of the committees and participants is on bmj.com 


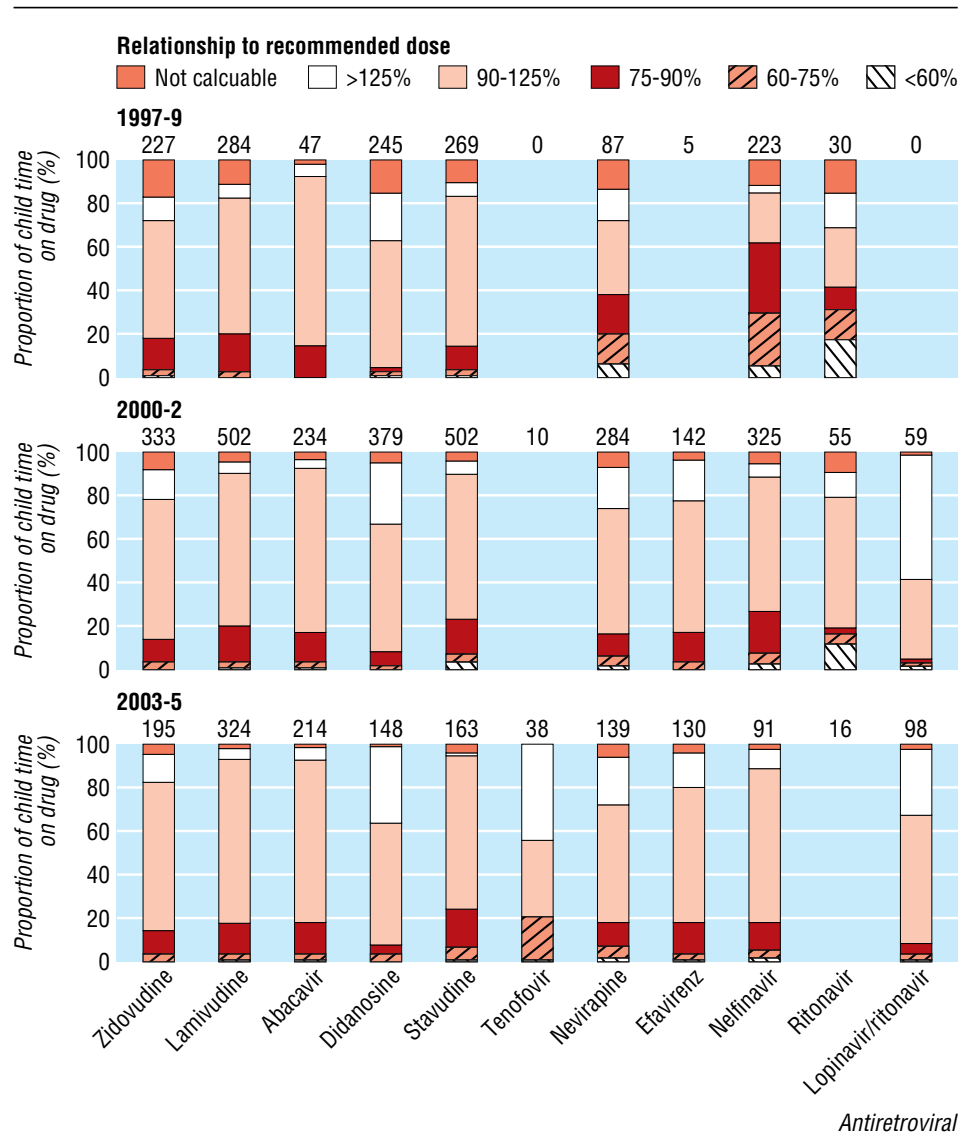

Fig 1 Proportion of child time taking common antiretrovirals for children aged 2-12 years, showing prescribed dose relative to the CRD. Numbers show total child years at risk; proportions not shown where fewer than 20 child years at risk

report any dose changes to CHIPS and data from eight rarely prescribed antiretrovirals. Three main classes of antiretrovirals were prescribed-nucleoside reverse transcriptase inhibitors, which commonly form the backbone of regimens; nonnucleoside reverse transcriptase inhibitors; and protease inhibitors.

\section{Weight (and height) measurements for dose calculations}

In total, 8907 weights (median 3.6 (interquartile range 2.7-4.6) per child per year) and 3660 antiretroviral dose changes (1.4 (0.6-2.7) per child per year) were reported from visits to clinic. $2788(76 \%)$ of changes to dose had weight reported on the same date; in $3321(91 \%)$ the most recent weight was within the preceding three months or subsequent six weeks (similarly for height).

Table 1 Demographics and disease history of the 615 children aged 2-12 years in the collaborative HIV paediatric study (CHIPS) who took antiretrovirals after 1 January 1997

\begin{tabular}{lc} 
Characteristic & No (\%) \\
\hline Female & $289(47 \%)$ \\
\hline Known to be vertically HIV-1 infected & $584(95 \%)$ \\
\hline Ethnic group: & \\
\hline Black & $445(72 \%)$ \\
\hline White & $90(15 \%)$ \\
\hline Mixed & $60(10 \%)$ \\
\hline Other/unknown & $20(3 \%)$ \\
\hline Received antiretrovirals before 1 January 1997 & $132(21 \%)$ \\
\hline
\end{tabular}

\section{Prescribed doses compared with CRD}

Doses standardised to weight or surface area varied widely across individual drugs and drug classes in all periods (fig 1). The proportion of time prescribed at less than $90 \%$ of the CRD varied between $6 \%$ and 62\%. Non-nucleoside reverse transcriptase inhibitors and protease inhibitors were underdosed more than nucleoside reverse transcriptase inhibitors, particularly in earlier time periods. Three specific patterns and dosing issues are highlighted below.

Underdosing related to changes in dosing recommendations after licensing

Dosing recommendations of the protease inhibitor nelfinavir were revised after licensing. Before important postlicensing pharmacokinetic data emerged to show that the original licensed

Table 2 Characteristics at the latest of 1 January 1997 or the start of treatment with antiretrovirals of children aged 2-12 years in the collaborative HIV paediatric study (CHIPS) who took antiretrovirals after 1 January 1997

\begin{tabular}{lll} 
Characteristic & No of children & $\begin{array}{l}\text { Median (interquartile } \\
\text { range) }\end{array}$ \\
\hline Age (years) & 615 & $4.8(2.2-8.1)$ \\
\hline Centers for Disease Control stage ${ }^{*}$ : & 615 & \\
\hline NA & & $231(38 \%)$ \\
\hline B & & $184 ; 30 \%$ \\
\hline C & 584 & $200 ; 33 \%$ \\
\hline CD4 cell percentage & 516 & $14(8-22)$ \\
\hline HIV-1 RNA $\left(\log _{10}(\right.$ copies/ml)) & $5.1(4.5-5.7)$ \\
\hline
\end{tabular}

*Two of three most severe categories only are presented. 


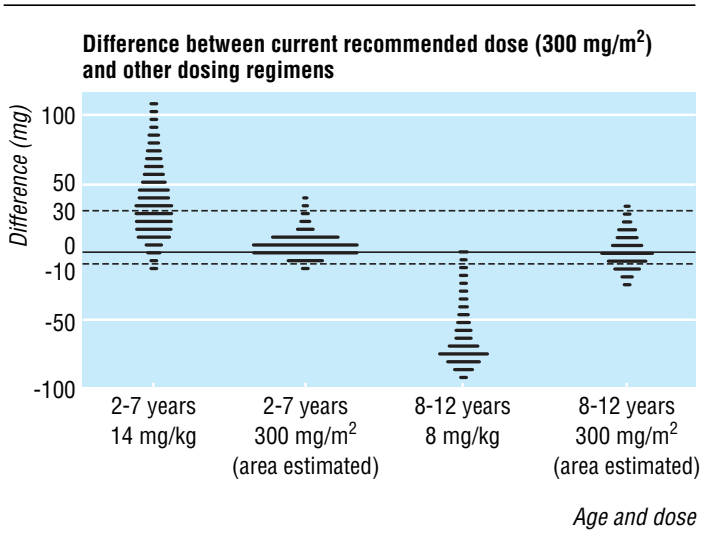

Fig 2 Recommended daily doses of nevirapine calculated from surface area or weight for each individual measurement of weight and height in CHIPS

dose (60-90 $\mathrm{mg} / \mathrm{kg}$ /day) was too low, ${ }^{13} 62 \%$ of child time taking nelfinavir (1997-9) was with doses less than 90\% of the CRD (110 $\mathrm{mg} / \mathrm{kg}$ /day). After this, underdosing fell to $26 \%$ in $2000-2$ and $18 \%$ in 2003-5.

Dose of antiretrovirals that have alternative dosage strategies (surface area or weight)

Original licensing studies for the non-nucleoside reverse transcriptase inhibitor nevirapine dosed by surface area (300-400 $\mathrm{mg} / \mathrm{m}^{2}$ a day), but also extrapolated for dose by weight and age $(14 \mathrm{mg} / \mathrm{kg} /$ day for ages 7 years and younger else 8 $\mathrm{mg} / \mathrm{kg} /$ day. The two strategies correspond poorly because of the abrupt change in dose at 8 years old, and because the relation between surface area and weight is not linear. For older children, the CRD based on weight is consistently less than the dose based on surface area (fig 2) whereas the reverse is true for younger children when the dose is above $200 \mathrm{mg}$ daily. Doses calculated from surface area that was estimated from weight, using common charts, were closer to doses calculated from actual surface area alone than doses calculated from weight and age.

Nevirapine doses related more closely to the CRD calculated by surface area than weight; $63 \%$ were within $90-125 \%$ of the CRD calculated by surface area compared with $48 \%$ by weight (similarly in each age group; fig 3). Over calendar time there was a clear trend of prescribing increasing doses, whether by surface area $(48 \%, 66 \%$, and $68 \%$ of doses prescribed were $90-125 \%$ of the CRD in 1997-9, 2000-2, and 2003-5 respectively) or by weight (39\%, $45 \%$, and $58 \%$, respectively).

Table 3 Exposure to antiretrovirals of 615 children aged 2-12 years after 1 January 1997 in the collaborative HIV paediatric study (CHIPS)*

\begin{tabular}{ll} 
Antiretroviral & Exposure (total child years) \\
\hline Zidovudine & 754 \\
\hline Lamivudine & 1111 \\
\hline Abacavir & 494 \\
\hline Didanosine & 773 \\
\hline Stavudine & 933 \\
\hline Tenofovir & 47 \\
\hline Nevirapine & 510 \\
\hline Efavirenz & 277 \\
\hline Nelfinavir & 638 \\
\hline Ritonavir & 102 \\
\hline Lopinavir/ritonavir & 156
\end{tabular}

*Additional child time exposed to other antiretrovirals: zalcitabine (42 child years);

emtricitabine (1); saquinavir (17); indinavir (25); amprenavir (14); and tipranavir, atazanavir, and enfuvirtide (all $<1$ child year)

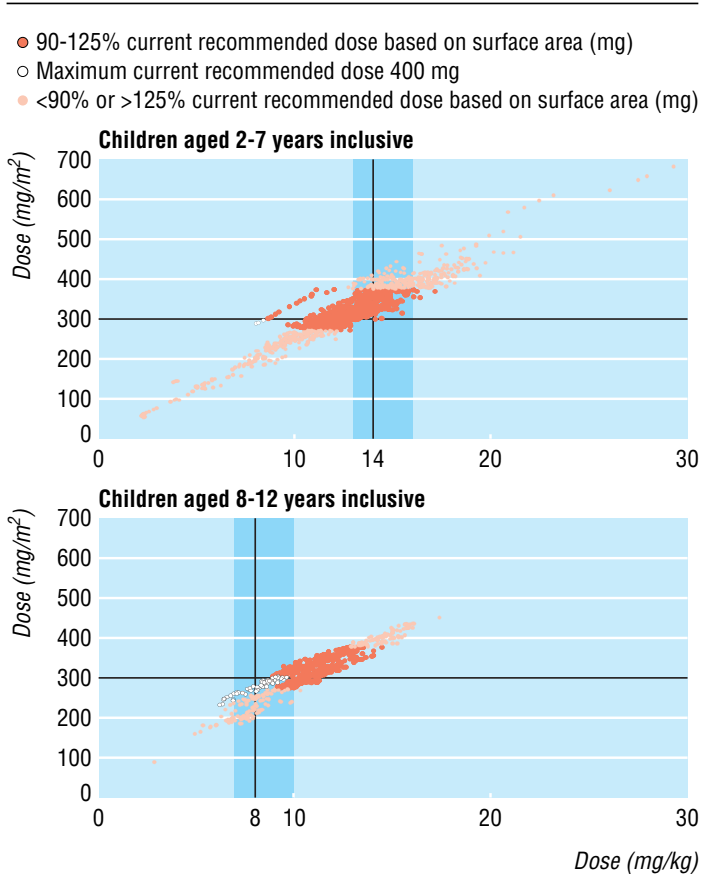

Fig 3 Nevirapine dosing corresponding to individual weight, height, and dose measurements for each child aged 2-12 years; hatched areas show doses corresponding to $90-125 \%$ current recommended dose based on dosing by weight; one child received a high dose of nevirapine in error for nine months aged 3 years

\section{Dose prescribing by weight bands}

Efavirenz, the alternative non-nucleoside reverse transcriptase inhibitor for children, was less commonly underdosed than nevirapine, and had more consistent dosing over calendar time $(16 \%$ and $17 \%$ of child time at less than $90 \%$ of the CRD by weight in 2000-2 and 2003-5, respectively, using an approximation of 12.5 $\mathrm{mg} / \mathrm{kg}$ to the complex formula ${ }^{14}$ used originally (see table A on bmj.com)). Manufacturer's guidelines recommend dosage by weight bands (fig 4), producing a tendency toward underdosing as a child's weight nears the top of each weight band, aggravated by children staying on a lower band despite weight increase.

\section{Predictors of underdosing}

Using multivariate binomial generalised estimating equations, we identified no consistent predictors of underdosing other than calendar year.

\section{Discussion}

Numerous studies have shown than combination antiretroviral treatment considerably improves prognosis for children infected with HIV $-1 .^{7{ }^{15} 16}$ Despite this, we found considerable underdosing of antiretrovirals in the UK and Ireland based on current best evidence. Some antiretrovirals were dosed suboptimally because of inadequate pharmacokinetic data at licensing ${ }^{12}{ }^{13}{ }^{17}$; other underdosing seems attributable to confusing and inconsistent dosage strategies or to failure to respond to growth, especially at the extremes of weight bands. Review of medical notes and pharmacy records at one centre (by EM and CW) identified failure to increase the dose with increases in height and weight or rounding down of doses as responsible for about half the underdosing. Limitations in formulation were responsible for about a third of underdosing (formulation data were not routinely available in CHIPS), and clinical indications or drug interactions for 


\section{Research}

the remainder. Legitimate dose reductions may have been required after toxicity, but these tend to occur less often than for adults who take antiretrovirals. ${ }^{18}$ We have planned further analyses to determine whether underdosing is related to therapeutic response, to evaluate whether the therapeutic index (the ratio of toxic dose to therapeutic dose) of individual drugs affects the extent of misprescribing or effectiveness.

Our study highlights important issues that apply throughout prescribing to children, particularly for other chronic diseases that need long term medication. Drug doses need regular adjustment as children grow, and failure to do so may reduce the benefits of treatment. Child friendly formulations are essential because existing tablet sizes designed for adults limit the precision with which doses can be given to children. Families with young children often prefer small pills (or dispersible, crushable, or scored tablets), rather than unpalatable suspensions with large volumes. HIV has useful surrogate markers of treatment efficacy (CD4 cells or percentage and HIV viral load). In conditions without equivalent markers inadequate dosing may go undetected until failure of treatment is seen clinically. Treatment of other chronic conditions, such as respiratory diseases of childhood, is largely prescribed off label and is also hampered by insufficient data on safety and efficacy in children. ${ }^{19}$ Our findings from this current study underline the importance of research on suitable formulations, dosages, and efficacy of drugs for children with acute and chronic diseases-for example, asthma. New dosing information that emerges after licensing is too slowly absorbed into clinical practice, even after publication. Where clinical and research networks are well established and integrated (for example, PENTA in Europe, www.pentatrials.org),
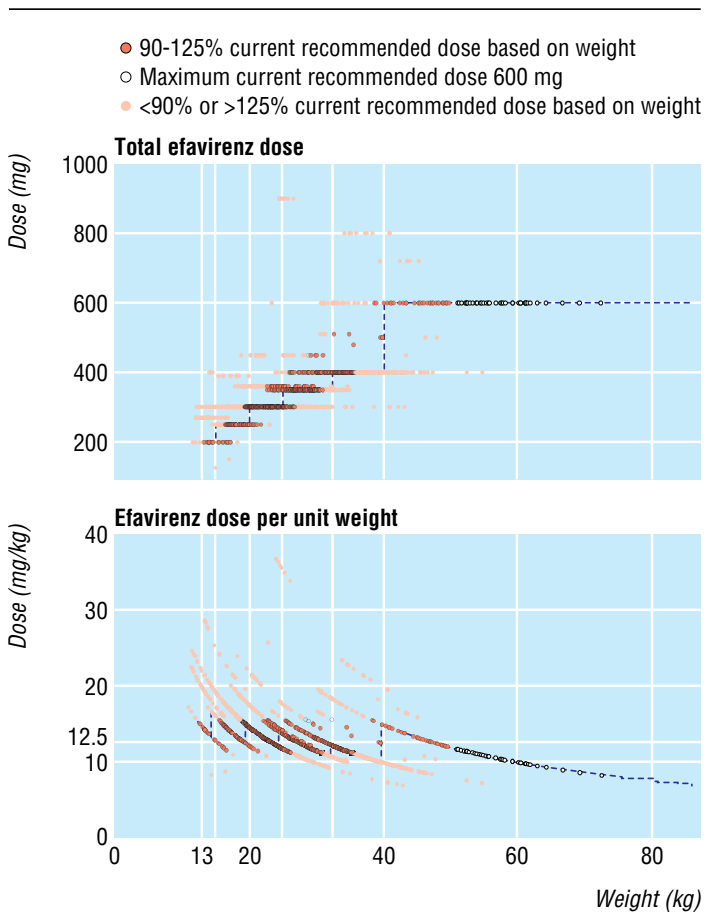

Fig 4 Dosing based on weight and weight bands for efavirenz. Dotted blue lines show licensed dose bands for capsules or tablets $(13-15 \mathrm{~kg}, 200 \mathrm{mg} ; 15-20 \mathrm{~kg}$, $250 \mathrm{mg} ; 20-25 \mathrm{~kg}, 300 \mathrm{mg} ; 25-32.5 \mathrm{~kg}, 350 \mathrm{mg} ; 32.5-40 \mathrm{~kg}, 400 \mathrm{mg}$; more than $40 \mathrm{~kg}, 600 \mathrm{mg}$ ). Capsules available are 50, 100, and $200 \mathrm{mg}$ plus a $600 \mathrm{mg}$ tablet. Efavirenz is also available as a liquid which is not bioequivalent with the capsule but requires higher doses $(15 \mathrm{mg} / \mathrm{kg})$. Formulation data were not collected in CHIPS over the study period. One child was taking high doses of efavirenz (>800 mg and $>30 \mathrm{mg} / \mathrm{kg}$ ) to allow for drug interactions

\section{What is already known on this topic}

The evidence base for prescribing drugs to children lacks sufficient pharmacokinetic and pharmacodynamic data

Adult doses are often extrapolated to children without taking account of potential differences in drug handling with age or dose requirements for effectiveness

Licensing data for paediatric dosing are often sparse, and subsequent studies may result in important changes to recommended doses

\section{What this study adds}

HIV infected UK and Irish children have been underdosed with antiretrovirals in the past nine years

Poor pharmacokinetic data at licensing results in incorrect drug dosing until important pharmacokinetic results emerge after licensing and inform revision of dosage recommendations

Guidelines stating alternative dosage strategies (by weight or surface area) for the same drug lead to different and inconsistent doses

Inadequate dosing also arises through failure to adjust for ongoing growth

early dissemination of important new research findings can promptly inform practice.

Drug manufacturers and expert guidelines use a variety of ways to calculate doses of paediatric drugs. For example, even the recently launched $B N F$ for Children ${ }^{20}$ gives amoxycillin dosing for pneumonia by age bands and for otitis media by weight. Phenoxymethylpenicillin and flucloxacillin are dosed by age bands, but the age bands differ markedly. Aciclovir is dosed both by weight and surface area. Such unexplained inconsistencies undermine the quality of paediatric prescribing perhaps worsening outcome at the individual and population level. In the absence of clear reasons for variations, simplification and unification of guidelines, with clarity from regulating bodies, would be preferable. Weight bands have certain advantages, particularly avoidance of calculation errors. ${ }^{21}$

Three key points emerge. Firstly, rigorous pharmacokinetic and pharmacodynamic data for children are needed before drug licensure. Secondly, effective formal systems for early appraisal, dissemination, and implementation of important modifications to treatment recommendations are needed universally. Thirdly, improved methods of pharmacovigilance are needed to monitor drug utilisation, efficacy and toxicity after drug licensing. The European Union ${ }^{22}$ and the United States have recently committed to promoting research specific to children's medicines while protecting children as participants in clinical trials. The UK Department of Health has launched the Medicines for Children Research Network (www.liv.ac.uk/morn), which aims to develop closer links between the drugs industry, regulators, families, and paediatricians, links that will be needed to meet the challenges of developing and manufacturing appropriate paediatric drugs (www.hivforum.org). Our study shows that, even for paediatric HIV - a new disease with rapid drug development and good dialogue between all these parties-antiretroviral dosing seems to have similar problems to the ones that antibiotics have always 
had. The Medicines for Children Research Network initiative to tackle these issues is timely.

Acknowledgments: The Collaborative HIV Paediatric Study (CHIPS) is a collaboration between the Medical Research Council Clinical Trials Unit, UK, and the National Study of HIV in Pregnancy and Childhood (NSHPC) at the Institute of Child Health, London. Committees and participants are on bmj.com

Contributors: All authors contributed to the writing up of the study. In addition, ENM, ASW, MS, and DMG contributed to the design; ENM and CW collected and collated data; and ASW carried out the analysis. All involvement was on behalf of the collaborative HIV paediatric study steering committee. DMG is guarantor.

Funding: CHIPS is funded by the London HIV Consortium and in the past has received additional support from Bristol-Myers Squibb, Boehringer Ingelheim, GlaxoSmithKline, Roche, Abbott, and Gilead

Competing interests: None declared.

Ethical approval: UK multicentre research ethics committee and relevant local research ethic committees.

1 Kauffman RE. Clinical trials in children: problems and pitfalls. Paediatr Drugs 2000;2:411-8.

2 Caldwell PHY, Murphy SB, Butow ON, Craig JC. Clinical trials in children. Lancet 2004;364:803-11.

3 Turner S, Longworth A, Nunn AJ, Choonara I. Unlicensed and off label drug use in paediatric wards: prospective study. BMJ 1998;316:343-5.

4 Medicines for children. EurActiv, 2004. www.euractiv.com/Article?tcmuri=tcm:29132015-16\&type $=$ LinksDossier (accessed 2 May 2006)

5 Food and Drug Administration. Pediatric drug development. Rockville, MD: FDA 2006. www.fda.gov/cder/pediatric/index.htm (accessed 2 May 2006)

6 MHRA/Department of Health strategy on medicines for children. www.mhra.gov.uk home/groups/pl-p/documents/websiteresources/con009667.pdf (accessed 2 May 2006).

7 Gibb DM, Duong T, Tookey PA, Sharland M, Tudor-Williams G, Novelli V, et al, on behalf of the National Study of HIV in Pregnancy and Childhood (NSHPC) and of the Collaborative HIV Paediatric Study (CHIPS). Decline in mortality, AIDS and hospital Collaborative HIV Paediatric Study (CHIPS). Decline in mortality, AIDS and hospital
admissions in perinatally HIV-1 infected children in the UK and Ireland. BMJ admissions in perinatally HIV-1 infected children in the UK and Ireland. BMJ 2003;327:1019-25.

8 Ades AE, Davison CF, Holland FJ, Gibb DM, Hudson CM, Nicholl A, et al. Vertically transmitted HIV infection in the British Isles. BMJ 1993;306:1296-9.

9 Sharland M, Blanche S, Castelli-Gattinara G, Ramos JT, Gibb DM, PENTA Steering Committee. PENTA guidelines for the use of antiretroviral therapy. HIV Medicin 2004;5:61-86. www.pentatrials.org/guidelines.htm

10 Sharland M, Gibb DM, Giaquinto C. Current evidence for the use of antretroviral therapy - a PENTA analysis. Eur J Paediatr 2000;159:649-56.

11 King JR, Kimberlin DW, Aldrovandi GM, Acosta EP. Antiretroviral pharmakcokinetics in the paediatric population: a review. Clin Pharmacokinet 2002;41:1115-33.
12 Litalien C, Faye A, Compagnucci A, Giaquinto C, Harper L, Gibb DM, et al Pharmacokinetics of nelfinavir and its active metabolite, hydroxy-tert-butylamine, in infants perinatally infected with human immunodeficiency virus type 1. Pediatr Infect Dis J 2003;22:48-55.

13 Krogstad P, Wiznia A, Luzuriaga K, Dankner W, Nielsen K, Gersten M, et al. Treatment of human immunodeficiency virus 1-infected children with the protease inhibitor nelfinavir mesylate. Clin Infect Dis 1999;28:1109-18.

14 Starr SE, Fletcher CV, Spector SA, Yong FH, Fenton T, Brundage RC, et al. Combination therapy with efavirenz, nevirapine and nucleoside reverse-transcriptase inhibitors in children infected with Human Immunodeficiency Virus type. $N$ Engl J Med in children infected

15 de Martino M, Tovo PA, Balducci M, Galli L, Gabiano C, Rezza G, et al. Reduction in mortality with availability of antiretroviral therapy for children with perinatal HIV-1 infection.JAMA 2000;284:190-7.

16 Gortmaker SL, Hughes, M, Cervia J, Brady M, Johnson GM, Seage GR, et al. Effect of combination therapy including protease inhibitors on mortality among children and adolescents infected with HIV-1. N Engl J Med 2001;345:1522-8.

17 Gatti G, Castelli-Gattinara G, Cruciani M, Bernardi S, De Pascalis CR, Pontali E, et al. Pharmacokinetics and pharmacodynamics of nelfinavir administered twice or thrice daily to human immunodeficiency virus type 1-infected children. Clin Infect Dis daily to human
2003;36:1476-82.

18 Alexanian A, Gibbons S, Lyall EGH, Walters S, Tudor-Williams G. Higher nevirapine doses correlate with improved outcomes in a paediatric population. $12^{\text {th }}$ Conference on Retroviruses and Opportunistic Infections. Boston, Ma, February 2005 [Abstract PL11.2].

19 Jong GW, Eland IA, Sturkenboom MC, Van Den Anker JN, Stricker BH. Unlicensed and off label prescription of drugs to children: population based cohort study. $B M J$ 2002;324:1313-4.

20 In: Costello I, ed. BNF for Children. London: Pharmaceutical Press, 2005.

21 Ponnet M, Frederix K, Petdachai W, Wilson D, Eksaengsri A, Zachariah R. A drug dosage table is a useful tool to facilitate prescription of antiretroviral drugs for Children in age table is a useful tool to facilitate presc
Thailand. Int J SDT AIDS 2005;16:420-6.

22 http://europa.eu.int/comm/enterprise/library/enterprise-europe/issue16/articles/ en/topic6en.htm

(Accepted 6 March 2006)

bmj.com 2006;332:1183

MRC Clinical Trials Unit, London NW1 2DA

Esse N Mensonspecialist registrar in paediatric infectious diseases

A Sarah Walkersenior statistician

Diana M Gibb

professor of epidemiology

St George's Hospital NHS Trust, London SW17 0QT

Mike Sharland consultant in paediatric infectious diseases

Carole Wellsconsultant paediatric HIV pharmacist

St Mary's Hospital NHS Trust, London W2 1NY

Gareth Tudor-Williamsconsultant in paediatric infectious diseases

E G Hermione Lyallconsultant in paediatric infectious diseases 